\title{
Traqueotomía en una unidad de tercer nivel del noroeste de México: descripción y análisis de casos
}

\author{
Juan Antonio Lugo Machado', Hugo Escobedo Delgado², Carlos Javier Mávita Corral ${ }^{3}$
}

\section{RESUMEN}

Objetivo: Describir las condiciones y características del procedimiento de traqueotomía en una unidad de tercer nivel de atención y analizar su asociación con el desenlace registrado.

Materiales y métodos: Estudio retrospectivo, observacional y descriptivo, que incluyó a los pacientes intervenidos por el Servicio de Otorrinolaringología del Centro Médico Nacional del Noroeste, Sonora, durante el período de un año agosto de 2014 al 2015. Revisión de expedientes clínicos de casos, incluyéndose 52 por considerarse completos. 57\% hombres; edad promedio: 56.2 años. Se empleó estadística descriptiva para caracterizar la distribución y las diferencias se evaluaron mediante prueba de ji cuadrado y T de Student.

Resultados: $71 \%$ se intervino en quirófano y $29 \%$ en la $\mathrm{UCl}$, siendo esta unidad quien indicó la operación en más de la mitad de los casos (51.92\%). La intubación prolongada fue la primera causa de traqueotomía (51\%). En 34,61\% se presentaron complicaciones y fueron en orden descendente: oclusión de cánula, enfisema periestomal, hemorragia, neumotórax bilateral, decanulación y fístula traqueoinnominada. Estas se hallaron significativamente asociadas con obesidad, intubación orotraqueal prolongada y uso de anestesia general. La mortalidad se presentó en 7,69\%.

Conclusiones: No se encontró diferencia significativa entre las complicaciones entre los pacientes de sala quirúrgica y cama de $\mathrm{UCl}$. La ventilación mecánica prolongada fue la principal indicación para traqueotomía. La decanulación fue la principal causa de complicación; asimismo el IMC es un factor de riesgo de complicaciones. La tasa de mortalidad fue elevada con $7.69 \%$ respecto a otros estudios.

Palabras clave: Traqueotomía; complicaciones; mortalidad.

\section{Tracheotomy in a tertiary health care unit in Northwestern Mexico: description and analysis of a series of cases}

\begin{abstract}
Objective: To describe the conditions and characteristics of the tracheostomy procedure in a tertiary health care unit and analyze its association with the recorded outcome.

Materials and methods: A retrospective, observational and descriptive study that included patients operated in the Otorhinolaryngology Unit of the Northwest National Medical Center, Sonora, from August 2014 to August 2015. Fifty-two (52) complete clinical records of consecutive series of cases were reviewed, out of which $57 \%$ were men with an average age of 56.2 years old. Descriptive statistics was used to characterize the distribution, and the differences were evaluated by chi-square test and Student's t-test.

Results: Seventy-one percent $(71 \%)$ of the patients underwent surgery in an operating room and $29 \%$ in the ICU. In the latter, more than half of the cases had a tracheostomy (51.92\%). Prolonged intubation was the main cause of $51 \%$ of the tracheostomy procedures. Complications reached $34.61 \%$ of the cases and included the following disorders in descending order: cannula occlusion, peristomal emphysema, hemorrhage, bilateral pneumothorax, decanulation and innominate fistula. These complications were significantly associated with obesity, prolonged endotracheal intubation and general anesthesia. Mortality occurred in $7.69 \%$ of the patients.

Conclusions: No significant difference was found between complications occurred in an operating room and the ICU. Prolonged mechanical ventilation was the primary indication for tracheotomy. Decannulation was the main cause of complication. Moreover, BMI was a risk factor for complications. The mortality rate was $7.69 \%$, which was more elevated compared to other studies.
\end{abstract}

Keywords: Tracheotomy; complications; mortality.

1. Médico Adscrito al Servicio de Otorrinolaringología.

2. Médico Residente de Otorrinolaringología. Hospital de Especialidades No. 2 "Luis Donaldo Colosio Murrieta", Centro Médico Nacional del Noroeste, Instituto Mexicano del Seguro Social, Ciudad Obregón. Sonora, México.

3. Médico Pasante del Servicio Social, Universidad de Sonora, Campus Cajeme, Ciudad Obregón. Sonora, México. 


\section{INTRODUCCIÓN}

Además de ser uno de los procedimientos más practicados en la actualidad, la traqueotomía es una de las intervenciones quirúrgicas más antiguas de las que se tenga registro (1). Las primeras representaciones datan de Egipto, aproximadamente 3600 años antes de la era común ${ }^{(2)}$, mientras que descripciones escritas pueden encontrarse en textos clásicos como el Rig Veda de la India (2000-1000 a. C.) y el Papiro de Ebers (1500 a. C.) (3). Referencias al respecto también fueron hechas en la antigua Grecia ${ }^{(4)}$, pero el primer registro científico fiable de traqueotomía exitosa fue realizado por el cirujano Antonio Musa Brasavola en 1546; sin embargo, un mayor interés se desarrolló durante el siglo XIX, a partir de la muerte por difteria del sobrino de Napoleón Bonaparte ${ }^{(5)}$. Posteriormente, la intervención sería empleada de manera extensiva durante las epidemias de poliomielitis ${ }^{(6)}$ y, a la fecha, es considerada como el manejo de elección para los pacientes que necesitan soporte ventilatorio mecánico prolongado o de protección de la vía aérea ${ }^{(7)}$.

Cada año, en Estados Unidos, alrededor de 800000 personas son sometidas a ventilación mecánica a causa de insuficiencia respiratoria aguda ${ }^{(8)}$ y hasta $34 \%$ de los pacientes que necesitan ventilación durante 48 horas reciben una traqueotomía ${ }^{(9)}$. Las indicaciones generales para la realización del procedimiento incluyen insuficiencia respiratoria aguda con la necesidad de ventilación mecánica prolongada, falla del retiro de asistencia ventilatoria, obstrucción de la vía aérea superior, vía aérea difícil y secreciones abundantes ${ }^{(7,10-13)}$. Hoy en día, la insuficiencia respiratoria aguda y la necesidad de ventilación mecánica prolongada representan dos tercios de todos los casos en que se indica la traqueotomía ${ }^{(14,15)}$.

La apertura de la tráquea tiene varias ventajas sobre la intubación translaríngea, incluyendo una mejor tolerancia del paciente, reducción de la irritación laríngea, mayor capacidad de comunicación, atención de enfermería más sencilla, así como reducción del espacio muerto y del trabajo respiratorio ${ }^{(7)}$. Las técnicas con que se practica la traqueotomía pueden dividirse en dos grandes grupos: la forma abierta (quirúrgica) y la percutánea. Esta última fue introducida en 1957 por Sheldon ${ }^{(16)}$ y en 1985 Ciaglia estableció las normas técnicas del procedimiento ${ }^{(17)}$. Ambas han experimentado variaciones a lo largo del tiempo, y la traqueotomía por dilatación percutánea ha ganado cada vez mayor popularidad en el paciente en estado crítico. La mayoría de los intensivistas están de acuerdo en que si un paciente requiere de ventilación mecánica durante más de 10-14 días, la traqueotomía está justificada y debe planificarse en condiciones óptimas ${ }^{(7,12,18,19)}$. No obstante, las indicaciones, riesgos, beneficios, tiempo y técnica del procedimiento siguen siendo controvertidos y dependen de la condición clínica de cada caso en particular ${ }^{(7)}$.

Las complicaciones de la traqueotomía se pueden clasificar en perioperatorias cuando ocurren en las primeras 24 horas, y en posoperatorias cuando ocurren después de ese tiempo. Las primeras se suelen presentar entre $2 \%$ y $6 \%$ de los casos, predominando la hemorragia, muerte, falsa vía, transfusión, extubación, neumotórax, enfisema, hipotensión e incapacidad de realizar la técnica. Las segundas comúnmente aparecen en el $9 \%-17 \%$ y entre otras destacan la hemorragia, el neumotórax, la obstrucción de la cánula, la decanulación accidental, los granulomas y la infección del sitio operatorio ${ }^{(20)}$.

Tras décadas de experiencia con la traqueotomía percutánea, aún existe un debate con respecto a su seguridad cuando se compara con la cirugía abierta en pacientes críticos. Cada año se publican nuevos reportes comparando ambas técnicas; los aspectos claves de la discusión giran en torno a la posibilidad de complicaciones, particularmente la mortalidad, hemorragia y tasas de infección ${ }^{(21)}$.

El objetivo de este estudio fue describir las condiciones y características del procedimiento de traqueotomía, así como analizar su relación con los desenlaces encontrados.

\section{MATERIALES Y MÉTODOS}

Estudio retrospectivo, descriptivo y correlacional efectuado a los pacientes intervenidos de traqueotomía en nuestro centro, durante el período de agosto de 2014 y agosto de 2015.

Criterios de inclusión: contar con un expediente clínico completo, que incluyera los siguientes datos: edad, sexo, índice de masa corporal, diagnóstico de ingreso, tiempo de intubación orotraqueal, lugar donde se ejecutó e indicaciones para la intervención, anestesia empleada, tipo de cánula colocada, complicaciones y desenlace.

Se hizo una revisión de los expedientes clínicos, a través de un muestreo no probabilístico por serie consecutiva de casos y la información se capturó en una matriz diseñada para este propósito. Los datos se analizaron con un paquete estadístico (SPSS para Windows ${ }^{\circledR}$, versión 22.0). Para las variables cualitativas se realizaron distribuciones de frecuencia; para las cuantitativas, medidas de resumen y tendencia central. Se examinó la relación entre la presencia de complicaciones y diversos antecedentes, a través de la prueba de $\mathrm{x} 2$ de Pearson y $\mathrm{T}$ de Student. Se consideró un valor de $\mathrm{p} \leq 0.05$ como estadísticamente significativo. 
Tabla 1. Traqueotomía en una unidad del noroeste de México. Tabla-análisis multivariado de la presencia de complicaciones y diversos factores.

\begin{tabular}{|c|c|c|c|}
\hline Variable & Odds Ratio & $95 \%$ Intervalo de confianza & $\mathbf{p}$ \\
\hline \multicolumn{4}{|l|}{ Sexo } \\
\hline Hombre vs. mujer & 0.875 & $0.276-2.773$ & 0.8205 \\
\hline \multicolumn{4}{|l|}{ Edad (años) } \\
\hline$\geq 50$ vs $<50$ & 1.126 & $0.327-4.847$ & 0.736 \\
\hline \multicolumn{4}{|l|}{ IMC (Kg/m2) } \\
\hline$\geq 25$ vs. $18.5-24.9$ & 2.613 & $0.807-8.463$ & 0.104 \\
\hline$\geq 30$ vs. $18.5-24.9$ & 5.175 & $1.331-20.108$ & $0.013^{*}$ \\
\hline \multicolumn{4}{|l|}{ Diagnóstico } \\
\hline Intubación orotraqueal prolongada vs. otros & 5.653 & $1.527-20.93$ & $0.006^{* *}$ \\
\hline Tumores y abcesos vs. otros & 0.122 & $0.014-1.046$ & $0.029^{*}$ \\
\hline Trauma y cuerpo extraño vs. otros & 0.482 & $0.089-2.609$ & 0.391 \\
\hline Tumores del sistema nervioso central vs. otros & 0.607 & $0.058-6.305$ & 0.674 \\
\hline \multicolumn{4}{|l|}{ Servicio } \\
\hline UCl vs. otros & 0.888 & $0.283-2.788$ & 0.840 \\
\hline Otorrinolaringología vs. otros & 3.75 & $0.896-15.691$ & 0.060 \\
\hline Terapia intermedia vs. otros & 3.826 & $0.745-19.648$ & 0.092 \\
\hline \multicolumn{4}{|c|}{ Tiempo de intubación orotraqueal previo a la cirugía } \\
\hline$\geq 10$ días vs. $<10$ días & 3.826 & $0.745-19.648$ & 0.092 \\
\hline \multicolumn{4}{|l|}{ Sitio donde se efectuó la intervención } \\
\hline Quirófano vs. UCl & 0.72 & $0.208-2.491$ & 0.603 \\
\hline \multicolumn{4}{|l|}{ Tipo de anestesia empleada } \\
\hline Balanceada general vs. local & 9.272 & $1.095-78.492$ & $0.018^{*}$ \\
\hline
\end{tabular}

Fuente: SNS: sistema nervioso central; UCI: unidad de cuidados intensivos. ${ }^{*} \mathrm{p}<0.05,{ }^{* *} \mathrm{p}<0.01,{ }^{* * *} \mathrm{p}<0.001$

\section{RESULTADOS}

De un total de 167 expedientes registrados con el procedimiento de traqueotomía, se excluyeron 118 por expedientes incompletos, incluyendo a 52 . El género predominante en esta serie fue el masculino, con 30 pacientes $(57,69 \%, 95 \%$ IC $44,19-70,13)$, mientras que el femenino aportó 22 (42,30\%, 95\% IC 29,87-55,81) (Tabla 2).
La media de edad fue 56.3 años ( $95 \%$ IC 51.06-61.50), con un rango de 18 a 89 , y el lugar donde mayoritariamente se ejecutó el evento quirúrgico fue la sala de quirófano, en 37 ocasiones $(71,15 \%$, 95\% IC $57,73-81,67)$, mientras que en las 15 restantes $(28,84 \%, 95 \%$ IC $18,33-42,27)$ se llevó a cabo en la Unidad de Cuidados Intensivos. En los 52 procedimientos se colocó cánula de traqueotomía Portex®.

Tabla 2. Características generales de la muestra y resultados del estudio

\begin{tabular}{|c|c|c|c|}
\hline Variable & Pacientes $(n=52)$ & Porcentaje (100\%) & $\begin{array}{c}95 \% \text { Intervalo de } \\
\text { confianza }\end{array}$ \\
\hline \multicolumn{4}{|l|}{ Sexo } \\
\hline Hombre & 30 & 57,59 & $44,19-70,13$ \\
\hline Mujer & 22 & 43,30 & $29,87-55,81$ \\
\hline \multicolumn{4}{|l|}{ Edad (años) } \\
\hline$<25$ & 6 & 11,53 & $5,40-22,97$ \\
\hline $25<50$ & 7 & 13,46 & $6,68-25,27$ \\
\hline $50-<75$ & 34 & 65,39 & $51,80-76,85$ \\
\hline$\geq 75$ & 5 & 9,62 & $4,18-20,61$ \\
\hline Media & \multirow{2}{*}{\multicolumn{2}{|c|}{56.3 años (rango: $18-89$}} & $51,06-61,50$ \\
\hline \multicolumn{2}{|l|}{ Índice de masa corporal $(\mathrm{Kg} / \mathrm{m} 2)$} & & \\
\hline$<18.5$ & 0 & 0 & $0-6,88$ \\
\hline $18.5-24.9$ & 31 & 59,61 & $46,07-71,84$ \\
\hline $25-29.9$ & 7 & 13,47 & $6,68-25,27$ \\
\hline$\geq 30$ & 14 & 26,92 & $16,77-40,25$ \\
\hline Media & \multicolumn{2}{|c|}{26.12 (rango: 18.9-40.9) } & $24,39-27-85$ \\
\hline
\end{tabular}




\begin{tabular}{|c|c|c|c|}
\hline \multicolumn{4}{|c|}{ Diagnóstico Intubación orotraqueal prolongada } \\
\hline DM + HAS + Cardiopatía & 2 & 3,84 & $1,06-12,98$ \\
\hline $\mathrm{DM}+\mathrm{HAS}+\mathrm{EVC}$ & 1 & 1,92 & $0,34-10,12$ \\
\hline DM + HAS + Neumonía & 1 & 1,92 & $0,34-10,12$ \\
\hline DM + HAS + EVC + Neumonía & 1 & 1,92 & $0,34-10,12$ \\
\hline DM + Cardiopatía & 4 & 7,69 & $3,03-18,17$ \\
\hline DM + Cardiopatía + EVC + Neumonía & 1 & 1,92 & $0,34-10,12$ \\
\hline DM + Cardiopatía + Neumonía & 2 & 3,84 & $1,06-12,98$ \\
\hline DM + EVC + Cardiopatía & 2 & 3,84 & $1,06-12,98$ \\
\hline HAS + Cardiopatía & 1 & 1,92 & $0,34-10,12$ \\
\hline HAS + Cardiopatía + EVC + Neumonía & 1 & 1,92 & $0,34-10,12$ \\
\hline HAS + EVC & 2 & 3,84 & $1,06-12,98$ \\
\hline HAS + EVC + Neumonía & 1 & 1,92 & $0,34-10,12$ \\
\hline EVC + Cardiopatía & 1 & 1,92 & $0,34-10,12$ \\
\hline EVC + Cardiopatía + Neumonía & 1 & 1,92 & $0,34-10,12$ \\
\hline Cetoacidosis diabética & 2 & 3,84 & $1,06-12,98$ \\
\hline Cetoacidosis diabética + HAS + Neumonía & 1 & 1,92 & $0,34-10,12$ \\
\hline Cetoacidosis diabética + Neumonía & 2 & 3,84 & $1,06-12,98$ \\
\hline Eclampsia & 1 & 1,92 & $0,34-10,12$ \\
\hline Total & 27 & 51,92 & $38,69-64,90$ \\
\hline \multicolumn{4}{|l|}{ Tumores/abscesos de cabeza y cuello } \\
\hline Cáncer laríngeo & 7 & 13,46 & $6,68-25,27$ \\
\hline Nasoangiofibroma & 2 & 3,84 & $1,06-12,98$ \\
\hline Tiroidectomía & 1 & 1,92 & $0,34-10,12$ \\
\hline Tumor de cuello & 1 & 1,92 & $0,34-10,12$ \\
\hline Absceso submaxilar & 1 & 1,92 & $0,34-10,12$ \\
\hline Total & 12 & 23,07 & $13,72-36,13$ \\
\hline \multicolumn{4}{|l|}{ Trauma y cuerpo extraño } \\
\hline TCE + Hemorragia subaracnoidea & 4 & 7,69 & $3,03-18,17$ \\
\hline Politraumatizado + TCE & 4 & 7,69 & $3,03-18,17$ \\
\hline Cuerpo extraño & 1 & 1,92 & $0,34-10,12$ \\
\hline Total & 9 & 17,30 & $9,38-29,73$ \\
\hline \multicolumn{4}{|l|}{ Tumores del sistema nervioso central } \\
\hline Total & 4 & 7,69 & $3,03-18,17$ \\
\hline \multicolumn{4}{|l|}{ Servicio de origen } \\
\hline Unidad de cuidados intensivos & 27 & 51,92 & $38,69-64,90$ \\
\hline Otorrinolaringología & 12 & 23,07 & $13,72-36,13$ \\
\hline Terapia intermedia & 10 & 19,23 & $10,80-31,90$ \\
\hline Urgencias & 1 & 1,92 & $0,34-10,12$ \\
\hline Medicina interna & 1 & 1,92 & $0,34-10,12$ \\
\hline Neurocirugía & 1 & 1,92 & $0,34-10,12$ \\
\hline \multicolumn{4}{|c|}{ Tiempo de intubación orotraqueal previo a la cirugía } \\
\hline$<10$ días & 13 & 25 & $15,23-38,21$ \\
\hline$\geq 10$ días & 39 & 75 & $61,79-84,77$ \\
\hline \multicolumn{4}{|l|}{ Sitio donde se efectúo la intervención } \\
\hline Quirófano & 37 & 71,10 & $57,73-81,67$ \\
\hline $\mathrm{UCl}$ & 15 & 28,9 & $18,33-42,27$ \\
\hline \multicolumn{4}{|l|}{ Tipo de anestesia empleada } \\
\hline Balanceada general & 39 & 75 & $61,79-84,77$ \\
\hline Local & 13 & 25 & $15,23-38,21$ \\
\hline \multicolumn{4}{|l|}{ Número de cánula empleada $(\mathrm{mm})$} \\
\hline 6 & 1 & 1,92 & $0,34-10,12$ \\
\hline 7 & 23 & 40,38 & $31,60-57,66$ \\
\hline 8 & 27 & 51,92 & $38,69-64,90$ \\
\hline 9 & 1 & 1,92 & $0,34-10,12$ \\
\hline \multicolumn{4}{|l|}{ Marca de la cánula } \\
\hline Portex® & 52 & 100 & $93,12-100$ \\
\hline Presentación de complicaciones & 18 & 34,61 & $23,15-48,20$ \\
\hline \multicolumn{4}{|l|}{ Tipo de complicaciones } \\
\hline Oclusión & 6 & 11,53 & $5,40-22,97$ \\
\hline Enfisema & 5 & 9,61 & $4,18-20,61$ \\
\hline Hemorragia & 3 & 5,76 & $1,98-15,64$ \\
\hline Decanulación & 1 & 1,92 & $0,34-10,12$ \\
\hline Fístula traqueoinnominada & 1 & 1,92 & $0,34-10,12$ \\
\hline Defunciones & 4 & 7,69 & $3,03-18,17$ \\
\hline
\end{tabular}

DM: Diabetes mellitus; HAS: hipertensión arterial sistémica; EVC: enfermedad vascular cerebral; UCI: unidad de cuidados intensivos 
El Servicio hospitalario donde más se indicó la intervención quirúrgica fue la Unidad de Cuidados Intensivos (UCI) con 27 $(51,92 \%$, 95\% IC 38,69-64,90), seguido de Otorrinolaringología con 12 (23,07\%, 95\% IC 13,72-36,13), Terapia Intermedia con $10(19,23 \%, 95 \%$ IC 10,80-31,90) y, por último, Medicina interna, Neurocirugía y Urgencias con un caso cada uno $(1,92$, $95 \%$ IC 0,34-10,12) (Figura 1).

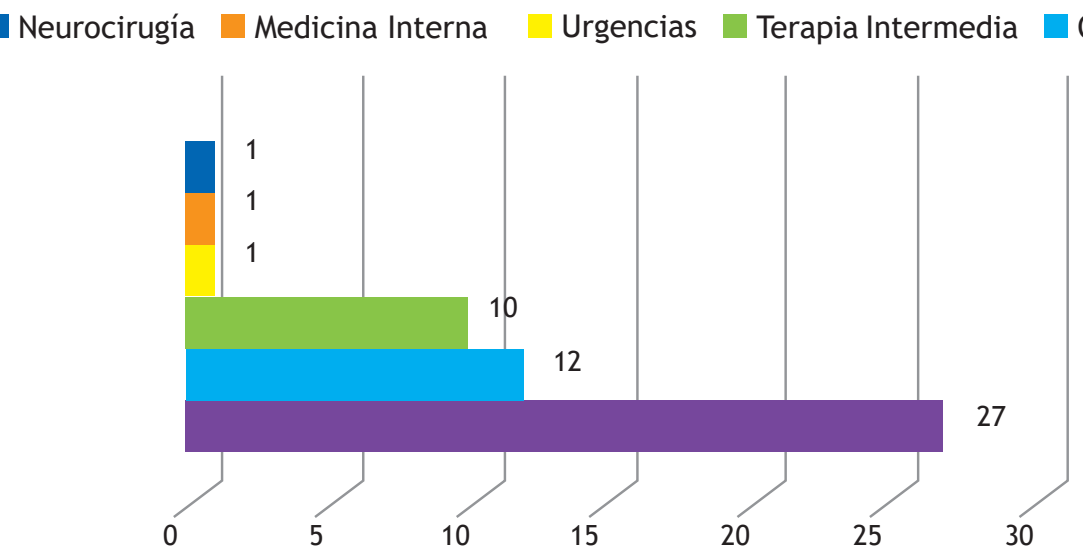

Figura 1. Distribución de los pacientes intervenidos de acuerdo a su servicio de origen

En relación al índice de masa corporal (IMC), de los 52 pacientes incluidos en el estudio, se encontró que 21 $(40,38 \%, 95 \%$ IC $28,16-53,93)$ presentaban un valor igual o superior a $25 \mathrm{Kg} / \mathrm{m} 2$, mientras que los $31(59,61 \%, 95 \%$ IC $46,07-71,84)$ restantes registraban uno inferior.

La principal indicación de traqueotomía en esta serie, fue la intubación orotraqueal prolongada con 27 casos, motivando la mitad de las intervenciones (51,92\%, 95 IC 38,69-64,90), la mayoría de las ocasiones por enfermedades metabólicas en pacientes de la $\mathrm{UCl}$. En segundo lugar se encontró a la obstrucción de la vía aérea por aumento de volumen del cuello, con 12 casos $(23,07 \%$, 95\% IC 13,72-36,13), seguida de trauma y cuerpo extraño en 9 casos $(17,30 \%$, 95\% IC 9,38-29,73) y, por último, tumores del sistema nervioso central con 4 casos $(7,69 \%$, 95\% IC 3,03-18,17) (Figura 2).

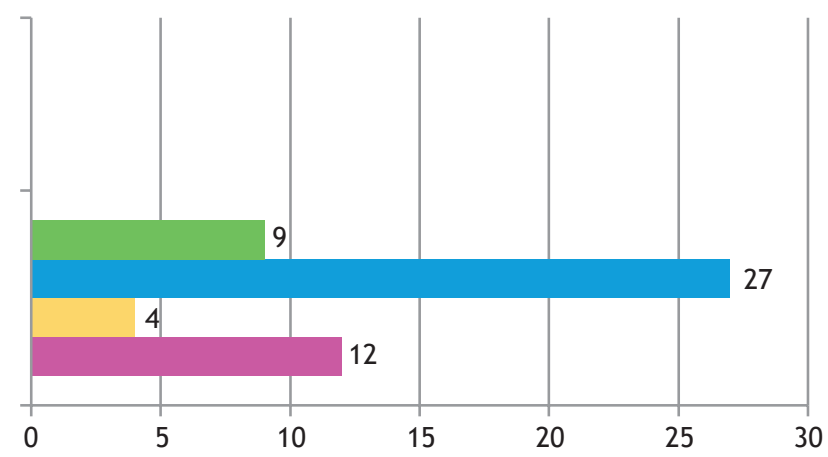

- Trauma y cuerpo extraño

- IOT prolongada (por enfermedad metabólica y eclampsia)

$\square$ Tumores del SNC

Tumores/abcesos de cabeza y cuello

Figura 2. Indicaciones de traqueotomía

El tipo predominante de anestesia empleada fue la de tipo general balanceada, que se aplicó en 39 pacientes $(75 \%$, $95 \%$ IC 61,79-84,77); en los 13 restantes, anestesia local con sedación $(25 \%$, $95 \%$ IC 15,23-38,21).
En cuanto a las complicaciones presentadas, del total de 52 pacientes incluidos en el estudio, 18 (34,61\%, $95 \%$ IC 23,15 $48,20)$ desarrollaron alguna. En frecuencia descendente, se hallaron: oclusión de cánula, enfisema periestomal, hemorragia, decanulación, fistula traqueoinnominada, neumotórax bilateral e infección (Figura 3). 


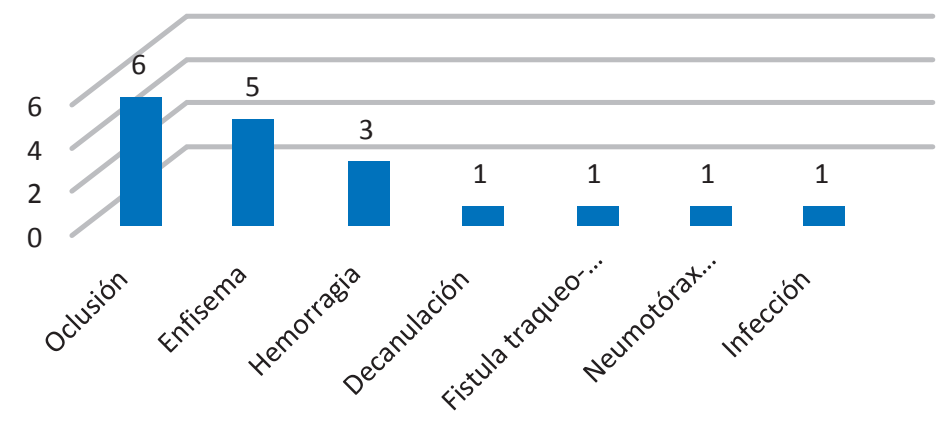

Figura 3. Complicaciones presentadas en 18 pacientes

Entre los 52 pacientes operados, ocurrieron 4 muertes que pueden relacionarse a la traqueotomía: dos casos por oclusión y falla ventilatoria, uno por neumotórax bilateral y otro secundario a una fistula traqueoinnominada que concluyó en choque hemorrágico. Conforme a lo anterior, la mortalidad fue de $7,69 \%(95 \%$ IC $3,03-18,17)$ en el periodo de un año (2014-2015).

Al efectuar un análisis correlacional entre la presencia de complicaciones y el resto de las variables recogidas en el estudio, se encontró una incidencia significativamente mayor con respecto al índice de masa corporal $(\mathrm{Kg} /$ $\mathrm{m} 2$ ) entre quienes contaban con $\geq 30$ vs. 18.5-24.9 (OR 5.175, 95\% IC 1.331-20.108, $\mathrm{p}=0.013$ ); también, entre los pacientes cuyo motivo de intervención fue la intubación orotraqueal prolongada en contraste al resto de diagnósticos (OR 5.653, 95\% IC 1.527-20.93, p=0.006); de igual forma, entre quienes recibieron anestesia balanceada general vs. anestesia local (OR 9.272, 95\% IC 1.095-78.492, $\mathrm{p}=0.018$ ). Por su parte, la frecuencia de complicaciones resultó menor en el grupo operado por tumores/abscesos de cabeza y cuello que en los demás (OR 0.122, 95\% IC 0.014-1.046, $p=0.029$ ).

\section{DISCUSIÓN}

En la presente serie de casos la traqueotomía fue practicada con más frecuencia en hombres que en mujeres: $57,69 \%$ vs. 42,30\%. En relación a lo anterior, los resultados reportados en la literatura son similares: Halum ${ }^{(13)}$, evidenció un ligero predominio del género masculino con $52 \%$ de los casos en un análisis multiinstitucional de 1175 pacientes en E.U.A., mientras que Johnson, en una revisión sistemática donde comparó las complicaciones en pacientes críticos de acuerdo a la técnica empleada, registró que (de 1199 casos) el $62,30 \%$ correspondía a varones ${ }^{(20)}$. En México, de 53 pacientes, Vázquez, observó $58,5 \%$ pertenecientes al género masculino ${ }^{(21)}$.

En relación a la edad media de los incluidos en el estudio (56,2 años), el resultado es semejante a lo presentado por otros autores: Halum encontró un promedio de 56,9 años ${ }^{(13)}$ mientras que en la revisión de Johnson, que incluyó 22 estudios, la edad media varió desde 32 hasta 72 años ${ }^{(20)}$. En Chile, Ortega ${ }^{(22)}$ halló una media de 48,9 años; por su parte, en México, Vázquez: 52,2 años ${ }^{(21)}$.
Respecto al sitio donde se efectuó la intervención, lo observado en esta serie: $71 \%$ en quirófano y $29 \%$ en la $\mathrm{UCl}$, es congruente a lo descrito por Ortega: $84,3 \%$ y $15,7 \%$, respectivamente ${ }^{(22)}$; por su parte, Halum ${ }^{(13)}$, encontró $71,8 \%$ y $22,9 \%$ (en $5,4 \%$ no se contó con el dato). No hubo diferencia significativa en las complicaciones con relación al lugar $(p>0.05)$. La traqueotomía ha pasado de ser un procedimiento exclusivo del quirófano a una práctica que cada vez se ejecuta con mayor frecuencia en la misma cama del enfermo en la UCI ${ }^{(21)}$.

Tal como lo señalado en otras publicaciones ${ }^{(22,28)}$, la principal indicación para traqueotomía fue la ventilación mecánica prolongada $(51,92 \%)$. En Italia, Vargas encontró dicha causa en el $58,8 \%$ de los casos, seguida por la necesidad de protección de la vía aérea en trastornos neurológicos/quirúrgicos/traumáticos en el $28,2 \%$, destete difícil o prolongado en $14,5 \%$, incapacidad para proteger la vía aérea en $6,9 \%$, dificultad para tragar y tos en $2,3 \%$ y la presencia de secreción abundante en $2,3 \%{ }^{(28)}$.

El tipo de anestesia empleada tampoco varió del reportado en la serie de Ortega, donde el 69,6\% de las intervenciones se hicieron con anestesia general y el 30,4\% restantes con anestesia local más sedación ${ }^{(22)}$; en el presente estudio los porcentajes fueron $75 \%$ y $25 \%$, respectivamente.

En cuanto a las complicaciones de la traqueotomía, en su estudio multicéntrico, Halum las registró en $14,1 \%$ de los $\operatorname{casos}^{(13)}$; Ortega, en $16,01 \%$ del total ${ }^{(22)}$; Vázquez, en $5,7 \%{ }^{(21)}$. Por su parte, en un estudio para comparar las complicaciones por traqueotomía quirúrgica contra la técnica dilatacional percutánea en pacientes de la $\mathrm{UCl}$, Yaghoobi consignó una tasa global de complicaciones del $40 \%$, equiparable entre ambos grupos, de las cuales la mayoría fueron leves y rápidamente solucionadas ${ }^{(7)}$. En la presente serie, las complicaciones ocurrieron en $34,61 \%$ de los casos ${ }^{(18 / 52)}$, sin embargo, esta discrepancia entre los resultados puede deberse a distintos factores, tales como: poblaciones diferentes, la habilidad y experiencia de los médicos adscritos o residentes, el enfoque de los procedimientos, requisitos para catalogar dichas complicaciones y criterios de inclusión a los estudios (como en este caso lo fue contar con un expediente clínico completo, en relación a lo cual podría suponerse que en 
caso de resultados adversos, el médico es más precavido y cuidadoso llenando los apartados del archivo).

En su estudio, Ortega describió que dentro de las complicaciones perioperatorias (presentes en $3,26 \%$ de los casos) la más frecuente fue el enfisema subcutáneo $(1,6 \%)$; las postoperatorias (ocurridas en $16,01 \%$ de las intervenciones) resultaron: infección del sitio operatorio $(7,5 \%)$, granuloma $(2,9 \%)$, hemorragia $(2,3 \%)$, fístula traqueocutánea $(1,6 \%)$, decanulación accidental $(0,6 \%)$, falsa vía $(0.3 \%)$ y obstrucción de la cánula $(0,3 \%)^{(22)}$. En la presente investigación predominó la oclusión de la cánula con 6 casos $(11,53 \%$ de las traqueotomías), seguida de enfisema periestomal con $5(9,61 \%)$, sangrado con $3(5,76 \%)$, así como infección local, decanulación, neumotórax y fistula traqueoinnominada con 1 caso $(1,92 \%)$ cada uno. Vargas, por su parte, encontró que las complicaciones tempranas más frecuentes fueron sangrado controlado con presión local y punción del tubo endotraqueal; las menos comunes, el neumotórax y el enfisema subcutáneo. Dentro de las tardías reportó también al sangrado de fácil control como la principal, además de infección del estoma, decanulación y hemorragia que requirió exploración ${ }^{(28)}$.

En esta serie de casos, el IMC resultó significativo para la presentación de complicaciones, lo cual coincide con lo reportado por otros autores, quienes han referido un riesgo significativamente mayor en los pacientes obesos ${ }^{(23-25)}$, sobre todo en el período intraoperatorio y postoperatorio temprano, especialmente en los que cuentan con un IMC igual o superior a $35^{(26,27)}$. Además de los mencionados, los pacientes con intubación orotraqueal prolongada tuvieron mayor riesgo de resultados adversos, lo cual ha sido señalado por otros autores, quienes reportaron 1.42 veces más probabilidad de tener una complicación postoperatoria tardía en individuos intubados por más de una semana; por su parte, la relación que se distinguió en este estudio entre resultados adversos y el tipo de anestesia empleado, difiere de los hallazgos en otras investigaciones, donde no se identificaron diferencias ${ }^{(13)}$.

La tasa de mortalidad asociada al procedimiento fue de $7,69 \%$, mientras que en el paciente con alguna de las complicaciones previamente mencionadas la cifra asciende a $22,22 \%$ (4 muertes entre 18 pacientes con alguna complicación de un total de 52 traqueotomías). Estos resultados difieren considerablemente de los reportados en E.U.A., donde Halum encontró que si bien existía una mortalidad por todas las causas de 22\% (generalmente motivada por la enfermedad subyacente), la tasa de mortalidad específica relacionada a una complicación de la traqueotomía era de 0,85\% ( $12 \%$ de los pacientes complicados) ${ }^{(13)}$, resultado similar a lo descrito por Shah en un estudio retrospectivo de cohorte que incluyó 113,653 operaciones, cuyos resultados mostraron una mortalidad de $19,2 \%$ sin importar la causa, $3,2 \%$ de complicaciones y $0,6 \%$ de muertes asociadas a la traqueotomía $(16,75 \%$ de los que presentaron alguna complicación) ${ }^{(29)}$.

Por su parte, Simon, en una revisión sistemática de la mortalidad secundaria a traqueotomía dilatacional percutánea, registró complicaciones letales en $0,17 \%$ de las intervenciones, equivalente a lo encontrado por Dennis, en un estudio donde evaluó más de 3000 procedimientos de traqueotomía percutánea: $0,16 \%$ de mortalidad ${ }^{(30,31)}$. En México, Vázquez observó una mortalidad general de 56,6\% en pacientes críticos con traqueotomía, pero sin separar las que ocurrieron asociadas al procedimiento ${ }^{(21)}$.

Si se excluye a la oclusión de la cánula como una complicación directa asociada al procedimiento y se atribuye, como ocurrió en la totalidad de los casos de esta serie, a los cuidados posteriormente brindados, la mortalidad específica se calcula en 3,84\% ( $95 \%$ IC 1,0612,98 ) de los pacientes intervenidos, mientras que entre quienes contaban con alguna complicación representa el $11,11 \%$ ( 2 muertes entre 18 pacientes complicados de un total de 52 operaciones). Así, la mortalidad es semejante a lo observado en pacientes pediátricos, en quienes se ha reportado de $0,05 \%$ hasta $6 \%$, sin embargo, continúa siendo alta ${ }^{(32,33)}$. De esta manera, se aprecia cómo los criterios para clasificar las complicaciones pueden influir en los resultados referidos.

Además de las limitaciones intrínsecas a un estudio retrospectivo, en esta investigación se observaron algunas otras como el contar con una muestra relativamente pequeña, haberse llevado a cabo en un solo centro hospitalario y excluir a más de dos tercios de los pacientes por no contar con un expediente completo.

En conclusión, no se encontró diferencia significativa entre las complicaciones entre los pacientes de sala quirúrgica y cama de $\mathrm{UCl}$; la ventilación mecánica prolongada fue la principal indicación para traqueotomía. La decanulación fue la principal causa de complicación; asimismo el IMC es fue un factor de riesgo de complicaciones. La tasa de mortalidad fue elevada con $7.69 \%$ respecto a otros estudios.

\section{REFERENCIAS BIBLIOGRÁFICAS}

1. Durbin C. Techniques for performing tracheostomy. Respir Care. 2005;50(4):488-96.

2. Pierson D. Tracheostomy from A to Z: historical context and current challenges. Respir Care. 2005;50(4):473-5.

3. McClelland R. Tracheostomy: its management and alternatives. Proceedings of the Royal Society of Medicine. 1972;65(4):401-4.

4. Goodall E. The story of tracheostomy. British Journal of Children's Diseases. 1934;31:167-76, 253-72.

5. Borman J, Davidson J. A history of tracheostomy: Si Spiritum Ducit Vivit (Cicero). Br J Anaesth. 1963;35:388-90.

6. Lassen H. A preliminary report on the 1952 epidemic of poliomyelitis in Copenhagen with special reference to the treatment of acute respiratory insufficiency. Lancet. 1953;1(6749):37-41.

7. Yaghoobi S, Kayalha H, Ghafouri R, Yazdi Z, Khezri M. Comparison of complications in percutaneous dilatational tracheostomy versus surgical tracheostomy. Glob J Health Sci. 2014;6(4):221-5.

8. Wunsch H, Linde-Zwirble W, Angus D, Hartman M, Milbrandt E, Kahn J. The epidemiology of mechanical ventilation use in the United States. Crit Care Med. 2010;38(10):1947-53. 
9. Quality of life after mechanized ventilation in the elderly study investigators. Two-month mortality and functional status of critically ill adult patients receiving prolonged mechanical ventilation. Chest. 2002;121(2):549-58.

10. De Leyn P, Bedert L, Delcroix M, Depuydt P, Lauwers G, Sokolov $Y$, et al. Tracheotomy: clinical review and guidelines. Eur J Cardiothorac Surg. 2007;32(3):412-21.

11. Rana S, Pendem S, Pogodzinski MS, Hubmayr RD, Gajic O. Tracheostomy in critically ill patients. Mayo Clin Proc. 2005;80(12):1632-8.

12. Martínez E, Berrazueta A, Romero J, Del Valle M, Fernández JA, Díaz C, et al. Analysis of a cohort of patients with indication of tracheostomy in intensive care medicine. Intensive Care Med Exp. 2015; 3(Suppl 1):1-2

13. Halum S, Ting J, Plowman E, Belafsky P, Harbarger C, Postma G, et al. A Multi Institutional Analysis of Tracheotomy Complications. A Multi-Institutional Analysis of Tracheotomy Complications. Laryngoscope. 2012;122(1):38-45.

14. Freeman B, Borecki I, Coopersmith C, Buchman T. Relationship between tracheostomy timing and duration of mechanical ventilation in critically ill patients. Crit Care Med. 2005;33(11):2513-20.

15. Frutos-Vivar F, Esteban A, Apezteguía C, Anzueto A, Nightingale P, González M, et al. Outcome of mechanically ventilated patients who require a tracheostomy. Crit Care Med. 2005;33(2):290-8.

16. Sheldon C, Pudenz R, Tichy F. Percutaneous tracheostomy. JAMA. 1957;165:2068-70.

17. Ciaglia P, Frisching R, Syniec C. Elective percutaneous dilatational tracheostomy. A new simple bedside procedure: preliminary report. Chest. 1985;87(6):715-9.

18. Durbin C. Tracheostomy: why, when, and how? Respir Care. 2010;55(8):1056-68.

19. Naser A, Celedón C, Neumann P, Ojeda JP. Revisión de la traqueostomía percutánea. Rev Otorrinolaringol Cir Cabeza Cuello. 2001;61:118-24.

20. Johnson-Obaseki S, Veljkovic A, Javidnia H. Complication rates of open surgical versus percutaneous tracheostomy in critically ill patients. Laryngoscope. 2016; 126(11): 2459-67

21. Vásquez-Revilla H, Revilla-Rodríguez E, Gualberto-González C, Terrazas V. Experiencia en la práctica de traqueostomía en el paciente críticamente enfermo en la Unidad de Cuidados Intensivos del Hospital Regional de Alta Especialidad de Oaxaca. Evid Med Invest Salud. 2015;8(4):167-71.

22. Ortega P, Ulloa J, Rivas L, Ulloa P. Experiencia clínica en traqueotomía abierta. Rev Otorrinolaringol Cir Cabeza Cuello. 2011;71:131-4.

23. El Solh A, Jaafar W. A comparative study of the complications of surgical tracheostomy in morbidly obese critically ill patients. Crit Care. 2007;11:R3.

24. McCormick T, Venn R. Recently published papers: tracheostomy: why rather than when? Obesity: does it matter? And stroke: diagnosis, thrombosis and prognosis. Crit Care. 2007;11(2):127.
25. Byhahn C, Lischke V, Meininger D, Halbig S, Westphal K. Perioperative complications during percutaneous tracheostomy in obese patients. Anaesthesia. 2005;60(1):125 .

26. Cordes S, Best A, Hiatt K. The impact of obesity on adult tracheostomy complication rate. Laryngoscope. 2015;125(1):105-10.

27. Marshall R, Haas P, Schweinfurth J, Replogle W. Tracheotomy outcomes in super obese patients. JAMA Otolaryngol Head Neck Surg. 2016; 142(8): 772-6.

28. Vargas M, Servillo G, Arditi E, Brunetti I, Pecunia I, Salami D, et al. Tracheostomy in Intensive Care Unit: a national survey in Italy. Minerva Anestesiol. 2013;79(2):156-64.

29. Shah R, Lander L, Berry J, Nussenbaum B, Merati A, Roberson D. Tracheotomy outcomes and complications: A national perspective. Laryngoscope. 2012;122(1):25-9.

30. Simon M, Metschke M, Braune S, Püschel K, Kluge S. Death after percutaneous dilatational tracheostomy: a systematic review and analysis of risk factors. Crit Care. 2013;17:R258.

31. Dennis B, Eckert M, Gunter O, Morris J, May A: Safety of bedside percutaneous tracheostomy in the critically ill: evaluation of more than 3,000 procedures. J Am Coll Surg. 2013;216(4):858-67.

32. Wilcox L, Weber B, Cunningham T, Baldassari C. Tracheostomy complications in institutionalized children with long-term tracheostomy and ventilator dependence. Otolaryngol Head Neck Surg. 2016;154(4):725-30.

33. Kremer B, Botos-Kremer A, Eckel H, Schlondorff G. Indications, complications, and surgical techniques for pediatric tracheostomies-An update. J Pediatr Surg. 2002;37(11):1556-62.

Fuentes de financiamiento:

Este artículo ha sido financiado por los autores.

\section{Conflictos de interés:}

Los autores declaran no tener ningún conflicto de interés.

\section{Correspondencia:}

Juan Antonio Lugo Machado

Dirección: Prolongación Hidalgo Bellavista, Cajeme 85130. Ciudad Obregón. Sonora, México.

Teléfono:+6441559891

Correo electrónico: otorrinox@gmail.com

Recibido: 26 de noviembre de 2016 Aprobado: 18 de marzo de 2017 